\title{
Apical Transportation and Centering Ability After Root Canal Filling Removal Using Reciprocating and Continuous Rotary Systems: A CBCT Study
}

\author{
Etienny da Silva Arruda ${ }^{1}$ Emílio Carlos Sponchiado-júnior ${ }^{1}$ Mariana Travi Pandolfo² \\ Fredson Márcio Acris de Carvalho ${ }^{3}$ Lucas da Fonseca Roberi Garcia ${ }^{2}$ André Augusto Franco Marques ${ }^{3}$
}

\footnotetext{
${ }^{1}$ School of Dentistry, Federal University of Amazonas, Manaus, Amazonas, Brazil

${ }^{2}$ Department of Dentistry, Endodontics Division, Health Sciences Center, Federal University of Santa Catarina, Florianópolis, Santa Catarina, Brazil

${ }^{3}$ Superior School of Health Sciences, State University of Amazonas,

Manaus, Amazonas, Brazil
}

Eur J Dent 2019;13:613-618
Address for correspondence André Augusto Franco Marques, DDS, MSc, $\mathrm{PhD}$, Superior School of Health Sciences, State University of Amazonas, 69050-030, Manaus, AM, Brazil (e-mail: andrendo1@hotmail.com).

\begin{abstract}
Keywords

- Apical transportation

- centering ability

- rotary systems

- reciprocating motion

Objective To evaluate the apical transportation and centering ability promoted by reciprocating and continuous rotary systems after root canal filling removal.

Materials and Methods After obturation, 40 mesial root canals of mandibular molars were distributed into four groups $(n=20)$ for filling material removal: PTU group-F2 instrument (25.08) of ProTaper Universal system; R25 group-R25 instrument (25.08) of Reciproc system; X2 group-X2 instrument (25.06) of ProTaper Next system and X3 group-X2 instrument (25.06) of ProTaper Next system, followed by X3 instrument (30.07). Cone-beam computed tomographic analysis was performed before and after filling material removal for acquisition of apical images. Apical transportation (AT) and its direction, and centering ability $(C A)$, were assessed using the equations $A T=(X 1-X 2)-(Y 1-Y 2)$ and $\mathrm{CA}=(\mathrm{X} 1-\mathrm{X} 2 / \mathrm{Y} 1-\mathrm{Y} 2$ or $\mathrm{Y} 1-\mathrm{Y} 2 / \mathrm{X} 1-\mathrm{X} 2)$, respectively. Data were submitted to the nonparametric Kruskal-Wallis and Dunn multiple comparison tests $(p<0.05)$ for statistical analysis. Results There was no statistically significant difference among groups for AT ( $p>0.05)$, with a tendency toward transportation in the distal direction. Also, there was no statistically significant difference among groups regarding CA ( $p>0.05)$.

Conclusions The different systems, including ProTaper Next, caused AT within the acceptable clinical limit after filling removal. In addition, none of the tested systems presented adequate $\mathrm{CA}$.
\end{abstract}

\section{Introduction}

The success rate of endodontic treatment has increased significantly over the last few years, mainly due to the development of new instruments and techniques that have led to safer and more efficient clinical performance. ${ }^{1}$ However, failures may occur at any stage of treatment, thereby compromising the desired result., ${ }^{1,2}$ Endodontic retreatment continues to be the primary alternative for resolution of these cases, in which the filling material occupying the root canal must be removed to enable new instrumentation and filling to be performed. ${ }^{3-5}$

Systems that use a reduced number of instruments for mechanical preparation of root canals are a contemporary trend, making the treatment faster and consequently diminishing both operator and patient fatigue. ${ }^{6,7}$ According to this concept, it would be expected that these systems would also be used for filling material removal, thereby obtaining success as relevant as that achieved when this procedure is performed by traditional methods. ${ }^{8-10}$ 
ProTaper Next system (Dentsply Maillefer, Ballaigues, Switzerland) is composed of five instruments-X1 (17.04), X2 (25.06), X3 (30.075), X4 (40.065), and X5 (50.06)-and it can perform mechanical preparation in a short period of time, in comparison with other rotary systems, due to the reduced number os instruments. ${ }^{5}$ Manufactured with M-Wire technology, the instruments have an innovative design, due to their rectangular cross-sectional shape that allows only two cutting edges to touch the root canal walls simultaneously, while the other two rotate freely, resulting in a rotary movement outside of their center of mass. ${ }^{1}$ This kinematics of movement generates less stress on the instruments, providing a corridor through which residues resulting from instrumentation may circulate. ${ }^{11,12}$

Due to these characteristics, studies have reported that ProTaper Next may be effective in performing endodontic retreatment. ${ }^{13,14}$ However, undesirable changes in the original trajectory of the root canal submitted to retreatment with this system still require more in-depth evaluations. ${ }^{15,16}$

Therefore, the purpose of this study was to evaluate the shaping ability (apical transportation and centering ability) of the ProTaper Next system after root canal retreatment in comparison with the ProTaper Universal (Dentsply Maillefer) and Reciproc (VDW, Munich, Germany) systems. The null hypothesis tested was that there would be no difference in the shaping ability of the systems.

\section{Materials and Methods}

\section{Sample Collection}

In this study, 40 recently extracted human mandibular molars were used after obtaining approval from the Research Ethics Committee (Protocol CAAE No. 52219315.1.0000.5020). Sample size calculation was performed with the aid of the Sealed Envelope software (Sealed Envelope Ltd., https://sealedenvelope. $\mathrm{com} /$ ), thereby obtaining a minimum number of 10 randomly selected root canals per experimental group for a power of $80 \%$ and type I error (level of significance) of 5\%. The teeth selected had completely formed roots and two independent mesial root canals. In addition, the selected teeth should have a mesial root with an angle of curvature of 30 degrees and radius of curvature of $10 \mathrm{~mm}$, which were calculated following the methods of Schneider ${ }^{17}$ and Pruett et al, ${ }^{18}$ respectively. Radiographic and tomographic examinations confirmed the anatomic findings necessary for inclusion or exclusion of the teeth from the final sample. Then, the teeth were disinfected in a $0.5 \%$ chloramine-T solution and stored in a receptacle containing distilled water, in which they were kept at $5^{\circ} \mathrm{C}$ until they were used.

Initially, the distal roots were sectioned with a diamond disc (KG Sorensen, Cotia, São Paulo, Brazil), and the mesial roots length standardized at $16 \mathrm{~mm}$. Coronal access was achieved with a spherical diamond burr No. 1015 (KG- Sorensen, São Paulo, SP, Brazil) mounted on a highspeed hand piece (Extra Torque 605C; Kavo, Joinville, SC, Brazil) under copious water cooling. A glide plane was created with an Endo Z burr (Dentsply Maillefer; Ballaigues, Switzerland) and a high-speed hand piece under copious water cooling.

\section{Biomechanical Preparation and Root Canal Filling}

With the purpose of standardizing the position of the roots during instrumentation, and later, when the tomographic examination was performed, the teeth were embedded in colorless self-polymerizing acrylic resin (Jet Classic, São Paulo, SP, Brazil), forming blocks that were afterward fitted onto a plastic base.

The mesial root canals were initially negotiated with sizes 10 and 15 K-type files (Dentsply Maillefer), establishing the working length at $15 \mathrm{~mm}$. Mechanical preparation was performed with the ProTaper Universal (Dentsply Maillefer) system, in accordance with the sequence of instruments recommended by the manufacturer, which are as follows: SX (19.04), S1 (18.02), S2 (20.04), and F1 (20.07). The instruments were coupled to a 6:1 contra-angle reducer (ENDO 6:1; Sirona Dental Systems GmbH, Bensheim, Germany), driven by an electric motor (X-SMART Plus, Dentsply-Maillefer) in rotary movement, at a speed of $300 \mathrm{rpm}$ and torque of $2 \mathrm{Ncm}$, in accordance with the manufacturer's instructions. The root canals were irrigated with $1 \mathrm{~mL}$ of $2.5 \%$ sodium hypochlorite solution (Rio Química; São José do Rio Preto, SP, Brazil) with the help of a disposable plastic syringe (Ultradent Products Inc., South Jordan, UT, United States) and yellow NaviTip needle (Ultradent Products Inc.), $3 \mathrm{~mm}$ short of the working length, after each gradual insertion of the instruments. At the end of the instrumentation, the excess of solution was aspirated with a Capillary Tip (Utradent Products Inc.), and the root canals were again irrigated with 2 mL of 17\% EDTA (Biodinâmica, Ibiporã, PR, Brazil) for 1 minute, followed by $2 \mathrm{~mL}$ of distilled water. Thereafter, the root canals were dried with sterile absorbent paper points (Dentsply-Maillefer) and filled with gutta-percha ProTaper F1 cones (Dentsply Maillefer) and AH Plus sealer (Dentsply, Petrópolis, RJ, Brazil) by means of a thermoplastification technique. Subsequently, the samples were stored in an oven (ECB1; Odontobrás, Ribeirão Preto, SP, Brazil) at $37^{\circ} \mathrm{C}$, and $100 \%$ relative humidity, for 15 days. At the end of this period, the samples were randomly distributed into four groups containing 10 mesial roots each, totaling 20 root canals $(n=20)$.

\section{Filling Material Removal}

Each experimental group was submitted to a different filling material removal protocol, as follows: PTU Group-instrument F2 (25.08) of the ProTaper Universal (Dentsply Maillefer) system in rotary movement at $300 \mathrm{rpm}$ and torque of $2 \mathrm{Ncm}$; R25 Group-instrument R25 (25.08) of the Reciproc(VDW) system in reciprocating movement; X2 Group-instrument X2 (25.06) of the ProTaper Next (Dentsply Maillefer) system in rotary movement at $300 \mathrm{rpm}$ and $2 \mathrm{Ncm}$ torque; and X3 Group-instrument X2 (25.06) of the ProTaper Next (Dentsply-Maillefer) system in the cervical and middle thirds, followed by the instrument X3 (30.07) for apical finishing, both in rotary movement at a speed of $300 \mathrm{rpm}$ and $2 \mathrm{Ncm}$ torque.

All the instruments were used in accordance with the recommendations of their respective manufacturers, in a 6:1 contra-angle reducer (ENDO 6:1, Sirona Dental Systems GmbH), driven by an electric motor (X-SMART Plus, Dentsply Maillefer). The instruments were gradually inserted into the root canals, with an in-and-out motion, light apical pressure, and 
amplitude of movement not exceeding $3 \mathrm{~mm}$. At each insertion and removal of the instruments from the root canal, they were cleaned with sterilized gauze. Each instrument was used for reinstrumentation of a maximum of two teeth, with exception of R25, which was used in only one tooth. The same irrigation protocol used during biomechanical preparation of the root canals was repeated during the reinstrumentation procedure. All the above-described procedures (initial preparation and retreatment) were performed by only one operator, specialist in endodontics.

\section{Apical Transportation}

Before biomechanical preparation, all teeth were submitted to the Cone Beam Computed Tomography (CBCT) assessment (CS 8100 3D, Carestream Health Inc., Rochester, NY, USA) for acquisition of apical third images, and analysis of the original configuration of the root canal trajectories. After initial preparation and filling, all samples went through the same process, and again after filling material removal, resulting in taking a total of three datasets of tomographic images. For this purpose, each set resin block/base set was adapted to a wax platform (that had low density, thus not interfering in performing tomography), with the mesial root parallel to the horizontal plane. Then, the samples were placed on the CBCT scanner, according to the following specifications: source of X-rays with value tension of 60 to $90 \mathrm{kVp}$, valve current 2 to $15 \mathrm{~mA}$, and focal point of $0.7 \mathrm{~mm}$.

For apical transportation analysis, images at $1 \mathrm{~mm}, 3 \mathrm{~mm}$, $5 \mathrm{~mm}$, and $7 \mathrm{~mm}$ of the apical third were selected, generating a total of eight axial images of each $1 \mathrm{~mm}$ for each canal. The transportation values were calculated by using the OsiriX Imaging software (OsiriX Imaging Software, http://dwww.osirix-viewer. com), in which the postreinstrumentation and prereinstrumentation diameters of the mesial root canal wall values were calculated by using the following equation: $\mathrm{AT}=(\mathrm{X} 1-\mathrm{X} 2)-(\mathrm{Y} 1-\mathrm{Y} 2)$.

The value of $\mathrm{X} 1$ corresponded to the mesial dentin wall thickness postinstrumentation; $\mathrm{X} 2$ was the mesial dentin wall thickness postreinstrumentation; Y1 was the distal dentin wall thickness postinstrumentation; and Y2 was the distal dentin wall thickness postreinstrumentation. ${ }^{19}$ When the value of AT was equal to zero, there was no transportation; when it presented a negative value, transportation occurred in the distal direction; and when the value was positive, transportation in the mesial direction was considered.

\section{Centering Ability}

The centering ability of the instruments was also calculated for the $1 \mathrm{~mm}, 3 \mathrm{~mm}, 5 \mathrm{~mm}$, and $7 \mathrm{~mm}$ of the apical third, compared with the values obtained during the transportation analysis, using the equation described by Gambill et a ${ }^{19}$ : $\mathrm{CA}=\mathrm{X} 1-\mathrm{X} 2 / \mathrm{Y} 1-\mathrm{Y} 2$ or $\mathrm{CA}=\mathrm{Y} 1-\mathrm{Y} 2 / \mathrm{X} 1-\mathrm{X} 2$. When the values were close to 1 (one), they indicated optimum centering ability; and when close to 0 (zero), they indicated lower ability of the instrument to maintain centralization in the root canal.

The images analysis and calculations for determining apical transportation and centering ability were performed in a blind manner by a single and previously calibrated examiner.

\section{Statistical Analysis}

The values obtained (apical transportation and centering ability) were analyzed as independent factors, considering both mesial canals and apical distance. The Kolmogorov-Smirnov test was initially applied to test the normality of the sample. Then, the data were submitted to the nonparametric Kruskal-Wallis and Dunn multiple comparison tests $(p<0.05)$. Statistical analysis was performed with the aid of GraphPad InStat software for Windows (GraphPad Software; La Jolla, CA, United States).

\section{Results}

\section{Apical Transportation}

The graphical representation ( $\mathrm{mm}$ ) for apical transportation and its direction may be visualized in - Fig. 1A, 1B.

There was no statistically significant difference among groups $(p>0.05)$, considering the different factors evaluated (apical distance and mesiobuccal or mesiolingual root canals). Furthermore, a total of 13 root canals, distributed throughout the four experimental groups $(\mathrm{PTU}=6 ; \mathrm{R} 25=3 ; \mathrm{X} 3=2 ; \mathrm{X} 2=2)$, had no apical transportation. Considering the direction of apical transportation, the highest rates occurred in the distal/inner direction $(57.8 \%)$ when compared with the outer mesial/outer direction (42.2\%).

\section{Centering Ability}

None of the instruments tested had complete centering ability $(=1.0)$. The group that had the centering ability closest to the ideal value was the group in which filling material removal was performed with instrument X2, followed by PTU, R25, and X3

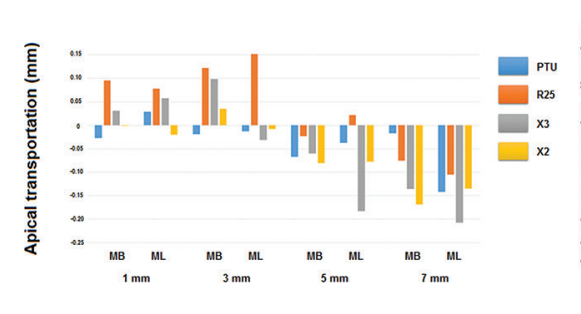

A

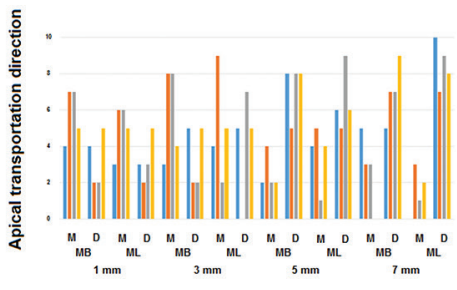

B
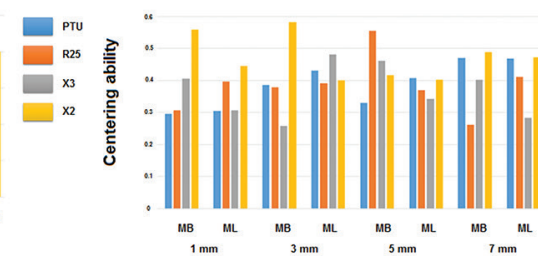

C

Fig. 1 Mean values of apical transportation and its direction, and centering ability. (A) Graphic representation (mm) of mean apical transportation values. (B) Graphic representation of apical transportation direction. (C) Graphic representation of mean centering ability values. There was no statistically significant difference among groups (Kruskal-Wallis, the Dunn multiple comparisons test, $p<0.05$ ). $n=20$. M-mesial; D-distal; MB-mesiobuccal; ML-mesiolingual. 
groups. However, there was no statistically significant difference among groups $(p>0.05)$ ( - Fig. 1C).

\section{Discussion}

The present study aimed to evaluate the shaping ability of the ProTaper Next system after filling material removal in comparison with the ProTaper Universal and Reciproc systems. Based on the results obtained, the null hypothesis was accepted, as the different systems presented similar performance with regard to apical transportation and centering ability after root canal retreatment.

Systems that use a reduced number of instruments for mechanical preparation have been constantly used for root canal retreatment. ${ }^{5}$ Therefore, changes in the original trajectory after root canal filling removal, using these systems, must also be evaluated. To the best of our knowledge, there is little information related to the apical transportation caused by these instruments and their centering ability when they are used for filling material removal. ${ }^{5}$ Just as the action of the instruments might promote undesirable changes in the original trajectory of the root canal during its preparation, ${ }^{2,6}$ the same might occur during its retreatment. ${ }^{5}$

The mesial canals of mandibular molars represent a challenge to clinicians, because they are generally atretic, and their roots invariably present an accentuated degree of curvature. ${ }^{20}$ For this reason, mandibular molars with two independent mesial canals, and a 30-degree angle of curvature-considered severe-were used in the present study. From a clinical point of view, it is relevant to simulate these conditions because these are the conditions that clinicians are frequently faced within their daily clinical practice. ${ }^{21}$

In this study, there was no significant difference among groups regarding transportation, irrespective of the apical portion or the mesial canal (buccal or lingual) assessed. These findings are in agreement with several other studies; ${ }^{22-24}$ however, the authors would like to point out that these studies were not performed in root canals submitted to retreatment, as it was performed in our study.

Fan et $\mathrm{al}^{25}$ have reported that apical transportation greater than $0.300 \mathrm{~mm}$ is clinically unacceptable, as it hinders the following stages of endodontic treatment, mainly root canal filling. Although apical transportation occurred in almost of all samples in the present study, the values ranged from $0.150 \mathrm{~mm}$ (mesial) to $-0.225 \mu \mathrm{m}$ (distal), ensuring favorable conditions to root canal obturation.

In a recent study, Nevares et $\mathrm{al}^{5}$ demonstrated that ProTaper Next and Reciproc systems had similar capacity for filling material removal during retreatment. These systems also presented equivalent levels of apical transportation, with clinically acceptable values. ${ }^{25}$ These results corroborated the findings of our study. However, in the study conducted by Nevares et $\mathrm{al},{ }^{5}$ reinstrumentation of root canals in the ProTaper Next group was initially performed with the instrument X3 (30.075) in the cervical and middle thirds, followed by the instrument X2 (25.06) for apical finishing. In the present study, in the X2 Group, there was no preflaring of the root canal (cervical and middle thirds enlargement) with the instrument X3. Only the instrument X2 was used until working length was reached. Whereas, in X3
Group, the instrument X2 was used in the cervical and middle thirds, followed by the instrument X3 for apical finishing. Therefore, it is valid to state that no enlargement of the cervical and middle thirds is needed when using the instrument X2 for root canal reinstrumentation.

Although apical preparation was performed with an instrument with larger diameter and taper (X3-30.075), the apical transportation was similar to the other groups. The same might be said in relation to the centering ability of the instruments. Larger diameter instruments tended to present greater difficulty in maintaining their long axis centralized with the root canal long axis. ${ }^{6}$ However, our findings demonstrated that the performance among the experimental groups was similar, even in the group where a larger instrument was used for apical preparation. Moreover, it is important to point out that none of the instruments tested demonstrated complete centering ability.

In addition, a discrete tendency toward apical transportation in the distal/inner direction was observed. Of the 320 points assessed in the root canals, 174 presented transportation in this direction. Differently from that observed in the present study, other research work reported a tendency toward higher transportation values in the mesial direction, as the distal wall of the root canal acts in the antifurca direction. ${ }^{21}$ This force presses the instrument against the mesial wall of the root canal, which is situated contrary to the curvature. ${ }^{23}$ However, we were able to note that the direction of transportation ranged according to the apical portion evaluated. In the first and third millimeters of the root canal, the trend toward transportation generally occurred in the mesial direction, whereas transportation occurred in the distal direction in the fifth and seventh millimeters. You et $\mathrm{al}^{2}$ demonstrated that this situation-variation in the direction of transportation within one and the same canal-may frequently occur, depending on the angle and radius of curvature of the root. In addition, several studies have reported that root canal preparation depends more on the root canal anatomy than the instruments used. ${ }^{2,6,24}$

Solvents such as eucalyptol and chloroform are frequently used in endodontic retreatment, because they promote gutta-percha dissolution, facilitating the action of instruments during root canal reinstrumentation. ${ }^{8,26}$ On the other hand, these substances are considered toxic, in addition to leading to formation of a softened gutta-percha-based residue that is difficult to remove, because it strongly adheres to the root canal walls, particularly in the apical portion. ${ }^{8}$ For this reason, no type of solvent was used before the action of the instruments in this study. The presence of residual gutta-percha in the apical portion may present difficulty in apical transportation analysis, thereby, compromising the accuracy of the results. ${ }^{5}$ Therefore, filling material removal in this study was performed according to the protocol of other recent studies that did not use solvents. ${ }^{4,14,27}$ Furthermore, it is worth emphasizing that this protocol was shown to be safe, since no instrument was fractured during reinstrumentation, even without presoftening of the gutta-percha. 


\section{Conclusions}

Despite the limitations of this in vitro study, the authors were able to affirm that all the systems tested, including ProTaper Next, presented clinically acceptable apical transportation values after filling material removal. With regard to their centering ability, none of the instruments were capable of maintaining their long axis centralized in the root canal, therefore, presenting similar performance.

\section{Author's Contributions}

Etienny da Silva Arruda contributed to data acquisition, data analysis, and manuscript preparation. Emílio Carlos Sponchiado-Júnior contributed to concept, study design, and manuscript review. Mariana Travi Pandolfo contributed to manuscript preparation and manuscript editing. Fredson Márcio Acris de Carvalho contributed to statistical analysis and manuscript review. Lucas da Fonseca Roberi Garcia contributed to manuscript preparation, manuscript editing, and manuscript review. André Augusto Franco Marques contributed to concept, study design, and manuscript review.

\section{Funding}

The authors would like to thank the "Fundação de Amparo à Pesquisa do Estado do Amazonas-FAPEAM" (Process $\left.\mathrm{N}^{0} 062.00649 / 2015\right)$ for the financial support.

\section{Conflict of Interest}

None declared.

\section{References}

1 Shen Y, Zhou HM, Zheng YF, Peng B, Haapasalo M. Current challenges and concepts of the thermomechanical treatment of nickel-titanium instruments. J Endod 2013;39(2):163-172

2 Costa EL, Sponchiado-Júnior EC, Garcia LFR, Marques AA. Effect of large instrument use on shaping ability and debris extrusion of rotary and reciprocating systems. J Investig Clin Dent 2018;9(1):e12289

3 Zuolo AS, Mello JE, Jr, Cunha RS, Zuolo ML, Bueno CE. Efficacy of reciprocating and rotary techniques for removing filling material during root canal retreatment. Int Endod J 2013;46(10):947-953

4 Üstün Y, Topçuoğlu HS, Düzgün S, Kesim B. The effect of reciprocation versus rotational movement on the incidence of root defects during retreatment procedures. Int Endod J 2015;48(10):952-958

5 Nevares G, de Albuquerque DS, Freire LG, et al. Efficacy of ProTaper NEXT compared with Reciproc in removing obturation material from severely curved root canals: a micro-computed tomography study. J Endod 2016;42(5):803-808

6 de Carvalho GM, Sponchiado Junior EC, Garrido AD, Lia RC, Garcia LdaF, Marques AA, , Garcia L da F, Marques AA. . Apical transportation, centering ability, and cleaning effectiveness of reciprocating single-file system associated with different glide path techniques. J Endod 2015;41(12):2045-2049

7 da Frota MF, Espir CG, Berbert FL, et al. Comparison of cyclic fatigue and torsional resistance in reciprocating single-file systems and continuous rotary instrumentation systems. J Oral Sci 2014;56(4):269-275

8 Fruchi LdeC, Ordinola-Zapata R, Cavenago BC. Hungaro Duarte MA, Bueno CE, De Martin AS. Efficacy of reciprocating instruments for removing filling material in curved canals obturated with a single-cone technique: a micro-computed tomographic analysis. J Endod 2014;40(7):1000-1004

9 Rios MdeA, Villela AM, Cunha RS, et al. Efficacy of 2 reciprocating systems compared with a rotary retreatment system for gutta-percha removal. J Endod 2014;40(4):543-546

10 de Souza PF, Goncalves LCO, Marques AA, Junior ECS, Garcia LDFR, de Carvalho FMA. Root canal retreatment using reciprocating and continuous rotary nickel-titanium instruments. Eur J Dent 2015;9(2):234-239

11 Wu H, Peng C, Bai Y, Hu X, Wang L, Li C. Shaping ability of ProTaper Universal, WaveOne and ProTaper Next in simulated L-shaped and S-shaped root canals. BMC Oral Health 2015;15:27

12 Zhao D, Shen Y, Peng B, Haapasalo M. Root canal preparation of mandibular molars with 3 nickel-titanium rotary instruments: a micro-computed tomographic study. J Endod 2014;40(11):1860-1864

13 Yılmaz K, Özyürek T. Apically extruded debris after retreatment procedure with Reciproc, ProTaper Next, and Twisted File Adaptive instruments. J Endod 2017;43(4):648-651

14 Martins MP, Duarte MA, Cavenago BC. Kato AS, da Silveira Bueno CE. Effectiveness of the ProTaper Next and Reciproc systems in removing root canal filling material with sonic or ultrasonic irrigation: A micro-computed tomographic study. J Endod 2017;43(3):467-471

15 Silva EJ, Orlowsky NB, Herrera DR, Machado R, Krebs RL, Coutinho-Filho TdeS. Effectiveness of rotatory and reciprocating movements in root canal filling material removal. Braz Oral Res 2015;29:1-6

16 Özyürek T, Demiryürek EÖ. Efficacy of different nickel-titanium instruments in removing gutta-percha during root canal retreatment. J Endod 2016;42(4):646-649

17 Schneider SW. A comparison of canal preparations in straight and curved root canals. Oral Surg Oral Med Oral Pathol 1971;32(2):271-275

18 Pruett JP, Clement DJ, Carnes DL Jr. Cyclic fatigue testing of nickel-titanium endodontic instruments. J Endod 1997;23(2):77-85

19 Gambill JM, Alder M, del Rio CE. Comparison of nickel-titanium and stainless steel hand-file instrumentation using computed tomography. J Endod 1996;22(7):369-375

20 D’Amario M, Baldi M, Petricca R, De Angelis F, El Abed R, D'Arcangelo $C$. Evaluation of a new nickel-titanium system to create the glide path in root canal preparation of curved canals. J Endod 2013;39(12):1581-1584

21 Gergi R, Osta N, Bourbouze G. Zgheib C, Arbab-Chirani R, Naaman A. Effects of three nickel titanium instrument systems on root canal geometry assessed by micro-computed tomography. Int Endod J 2015;48(2):162-170

22 Gagliardi J, Versiani MA, de Sousa-Neto MD, Plazas-Garzon A, Basrani B. Evaluation of the shaping characteristics of ProTaper Gold, ProTaper NEXT, and ProTaper Universal in curved canals. J Endod 2015;41(10):1718-1724

23 Zanesco C, Só MV, Schmidt S, Fontanella VR, Grazziotin-Soares $\mathrm{R}$, Barletta FB. Apical transportation, centering ratio, and volume increase after manual, rotary, and reciprocating instrumentation in curved root canals: analysis by micro-computed tomographic and digital subtraction radiography. J Endod 2017;43(3):486-490

24 Elnaghy AM, Al-Dharrab AA, Abbas HM, Elsaka SE. Evaluation of root canal transportation, centering ratio, and remaining dentin thickness of TRUShape and ProTaper Next systems in curved root canals using micro-computed tomography. Quintessence Int 2017;48(1):27-32 
25 Fan B, Wu MK, Wesselink PR. Leakage along warm guttapercha fillings in the apical canals of curved roots. Endod Dent Traumatol 2000;16(1):29-33

26 Agrafioti A, Koursoumis AD, Kontakiotis EG. Re-establishing apical patency after obturation with Gutta-percha and two novel calcium silicate-based sealers. Eur J Dent 2015;9(4):457-461
27 Gorduysus MO, Al-Rubai H, Salman B. Al Saady D, Al-Dagistani $\mathrm{H}$, Muftuoglu S. Using erbium-doped yttrium aluminum garnet laser irradiation in different energy output levels versus ultrasonic in removal of root canal filling materials in endodontic retreatment. Eur J Dent 2017;11(3):281-286 